\title{
How Many Measurement Times for Probe Reaction Time could be Yielded Optimum and Reliability?
}

J. Phys. Ther. Sci.

20: 91-95, 2008

\author{
ChUn Ying Hu ${ }^{1,2)}$, Ming HuO ${ }^{3)}$, Hitoshi MaruYAmA ${ }^{3)}$ \\ ${ }^{1)}$ Graduate School of International University of Health and Welfare \\ ${ }^{2)}$ Chiba Rehabilitation Research Center \\ ${ }^{3)}$ Department of Physical Therapy, International University of Health and Welfare: 2600-1 \\ Kitakanemaru, Ohtawara City, Tochigi 324-8501, Japan. TEL +81 287-24-3000
}

\begin{abstract}
Purpose] This study examined the reliability of measurement of the probe reaction time (P-RT) in different types of repetitive movement. Also the study tried to determine the optimal number of times PRT should be taken to yield reliable measurements. [Subjects] Ten subjects ( 7 males, 3 females) measured on two consecutive days. [Methods] Six different types of movements were tested including rest sitting position, marking time on sitting, marking time on standing, walking, tapping with the non-dominant hand at $1 \mathrm{~Hz}$ and $4 \mathrm{~Hz}$. Simple reaction time (SRT) and P-RT movements were measured 10 times. [Results] The results show that the P-RT of marking time on sitting position, marking time on standing, walking, tapping with the non-dominant hand at $1 \mathrm{~Hz}$ and tapping $4 \mathrm{~Hz}$ had high interclass correlation coefficients. Further analysis showed that there were significant correlations among the six different kinds of movements, and that the 3rd time measurement could yield the optimal results. [Conclusion] In the clinical field, especially for the patients who are not able to walk or stand and weak people, the number of times of testing for P-RT could be reduced for each the six movements.
\end{abstract}

Key words: Reliability, Probe Reaction Time, Repetitive Movement

(This article was submitted Oct. 11, 2007, and was accepted Nov. 28, 2007)

\section{INTRODUCTION}

There are many studies reporting the measurement of reaction time (RT) and the probe reaction time (P-RT). However, there is no study to date on the reliability of measurement of RT and P$\mathrm{RT}$ and the optimal number of measurement times which should be taken for subjects or patients.

In one study, a physiological profile approach to falls risk assessment and prevention was assessed in milliseconds using a hand-held electronic timer and light as the stimulus, and depression of a switch by the finger and foot as the responder ${ }^{1)}$. In his study, the RT was measured after 5 practice trials which were followed by 10 experimental measurements.
In another study by Kimberly, RT was measured by pushing a hand-held button as quickly as possible in response to auditory tones 10 times $^{2}$. In yet another study, William performed RT 24 times (6 times per condition) on participants who were required to respond to an auditory cue as quickly as possible, by loudly saying the word "top" 3 . Kurosawa performed measurements of P-RT for different movements 10 times on subjects, regardless of the type of movements ${ }^{4}$. In a previous study, P-RT was measured 10 times with subjects standing ${ }^{5}$. In another previous study, P-RT was measured 5 times with subjects standing and then 15 times during a stepping movement, with groups of 5 measurements randomly assigned to the left and right swing phase, 
and the double stance phase, respectively ${ }^{6}$.

Previously, measurements of RT and P-RT have been performed a number of times, ranging from 5 to 10 or more in order to get optimal and effective results. However, measurements can only be done in laboratory settings, as most of the measurement procedures are difficult to use widely because of the restrictions due to equipment and the environment for testing. Certain flaws are found in these experiments. The contributing rates are low unless very expensive equipment is available. As a result, consistency of measurement in RT and P-RT could not be achieved in these experiments. If the test is performed too many times, it may induce fatigue in the participants to the extent that results lose accuracy during the experiment. If the number of the test movements performed is insufficient, the reliability and validity of the results may be influenced.

$\mathrm{RT}$ is different when subjects perform different types of repetitive movements. In psychology, RT is thought to be a limitation in capacity of processing information from the environment. For instance, if a different task is applied to a subject when she/he is performing a movement task, the two tasks performed at the same time are defined as a dual task. If the main task is comparatively simple, a relatively large amount of attention can be allocated to the second task. This makes it possible to perform the second task relatively quickly. Thus, it is interpreted that a lot of attention can be resources allocated to the second task. Therefore, if the second task (simple reaction time) is demanded during movement task enforcement and the RT to the dual task is relatively short, it implies that the main task is being performed automatically. This method is called probe reaction time. This study method is recommended in movement-related research when trying to identify the attention for performing the main task. Measuring phonatory reaction time is particularly recommended in such studies because it can sensitively recognize a slight change in attention demanded in voluntary movements ${ }^{7)}$. In this study, we report on P-RT measurement from the 10 times, the interclass correlation coefficient (ICC) of each number of time to decide how many number of measurement times of the P-RT should be tested in different types of movements. We further discuss the reliability and reproducibility of measurements in P-RT in each movement.

\section{SUBJECTS AND METHODS}

\section{Subjects}

Ten healthy people with a mean age of $27.0 \pm 7.5$ years, and a mean height of $170.8 \pm 9.5 \mathrm{~cm}$, a mean body weight of $65.1 \pm 11.1 \mathrm{~kg}$ were recruited for this study. All the subjects were able to perform activities of daily life independently. All the subjects gave their consent to participate in this study.

\section{Methods}

To examine the reliability of P-RT in the repetitive movements or actions, 10 subjects were selected at random from among the students and staffs of our university. The tests were performed and the retests were implemented on the following day. We evaluated SRT in rest sitting position; and $\mathrm{P}-\mathrm{RT}$ in marking time on sitting, marking time on standing; walking; and tapping with the nondominant hand at $1 \mathrm{~Hz}$ and $4 \mathrm{~Hz}$ in synchrony with beats of a metronome. The speed of marking time on sitting, marking time on standing and walking were self-determined. RT was measured 10 times.

A digital audio player/recorder (Rio.Japan) was used for the auditory stimulus and also as the recording device. The auditory stimulus file was prepared on a computer beforehand. The file was edited as a series of 16 warning signal and auditory stimulus $(50 \mathrm{~ms})$ sets using a personal computer overtone opinion processing software of DigionSound5 (Digion). The file was downloaded to the digital audio player/recorder, and the digital audio player/recorder was connected to a portable speaker, so as to form an auditory cue box. The interval between warning signals and auditory stimulus was completely randomized within the range of 2-3 seconds.

A compact advanced rhythm trainer/digital metronome (Beatlab-1) was used for creating the rhythm at $1 \mathrm{~Hz}$ and $4 \mathrm{~Hz}$ when subjects tapped with the non-dominant hand.

The experiments were conducted to indicate overall attention ability. The movements were used to measure the simple phonatory reaction time to an auditory stimulation. The auditory cue box was attached to the abdominal region of the subjects to measure the P-RT. The subjects were required to respond to the auditory cue by loudly saying the word "Pa" as quickly as possible. The sound "Pa" was recorded by the digital audio player/recorder 
Table 1. Mean and SD of 3rd and 10th Time During Each Movement $(n=10)$

\begin{tabular}{cccc}
\hline $\begin{array}{c}\text { Types of repetitive } \\
\text { movement }\end{array}$ & $\begin{array}{c}\text { Times of } \\
\text { measurement }\end{array}$ & $\begin{array}{c}\text { First measurement } \\
(\mathrm{msec})\end{array}$ & $\begin{array}{c}\text { Second measurement } \\
(\mathrm{msec})\end{array}$ \\
\hline SRT & 3rd & $291.2 \pm 60.0$ & $299.3 \pm 75.3$ \\
& 10th & $292.5 \pm 75.8$ & $297.0 \pm 74.7$ \\
MTSi & 3rd & $299.5 \pm 65.6$ & $275.4 \pm 59.8$ \\
& 10th & $329.2 \pm 90.4$ & $321.0 \pm 72.9$ \\
MTSt & 3rd & $274.4 \pm 63.4$ & $277.0 \pm 61.5$ \\
& 10th & $294.4 \pm 64.4$ & $284.7 \pm 57.1$ \\
W & 3rd & $296.2 \pm 69.9$ & $258.2 \pm 76.1$ \\
& 10th & $281.2 \pm 62.4$ & $278.1 \pm 67.0$ \\
1Hz & 3rd & $333.5 \pm 93.0$ & $321.0 \pm 78.7$ \\
& 10th & $300.3 \pm 68.6$ & $280.1 \pm 83.0$ \\
$4 \mathrm{~Hz}$ & 3rd & $304.4 \pm 57.8$ & $287.2 \pm 52.2$ \\
& 10th & $297.8 \pm 52.0$ & $284.7 \pm 53.2$
\end{tabular}

SRT $=$ Rest Sitting Position, MTSi $=$ Marking Time on Sitting, MTSt $=$ Marking Time on Standing, $\mathrm{W}=$ Walking, $1 \mathrm{~Hz}=$ Tapping at $1 \mathrm{~Hz}, 4 \mathrm{~Hz}=$ Tapping at $4 \mathrm{~Hz}$.

hanging on the subjects' waist. RT was measured as the interval between the auditory stimulus of "Pi" and the utterance of "Pa".

Prior to the experiment, the subjects were told of what would be done in the experiment, and they made trial exercises to familiarize themselves with the procedures. In the actual experiment, for SRT, the subjects were asked to sit quietly on a chair to respond to the auditory cue. For P-RT, the subjects were asked to mark time while sitting in the chair, mark time while standing, and while walking, as well as tapping with the non-dominant hand in synchrony with beats of the metronome at the frequencies of $1 \mathrm{~Hz}$ and $4 \mathrm{~Hz}$. The speed of marking time on sitting, marking time on standing and walking were self-determined by the subject. P-RT was measured 10 times continuously in the different kinds of repetitive movements respectively. Data was input into a personal computer, and the DigionSound5 sound-processing software was used for the analysis.

In order to assess the reliability of measurement times and the reliability of re-test of P-RT, ICCs were used. The data were analyzed using SPSS Ver.12.0 for Windows. Analysis of variance was used to examine the experimental times for the six movements. Post hoc comparisons for both conditional movements and experimental times were performed using the Scheffe test. The level of significance was $\mathrm{p}<0.05$.

\section{RESULTS}

The means and standard deviations of the 3rd and the 10th measurement times of the six movements are shown in Table1. Main effects for the movements and experimental times were not found, and there was no significant difference within the movement conditions and experimental times.

The ICCs of different types of repetitive movement are shown in Table 2. The ICC of rest sitting position ranged from 0.52 for the $1 \mathrm{st}$ measurement time to 0.98 for the 10 th measurement time. An ICC of 0.92 for rest sitting position was achieved by the 4 th measurement time. The ICC of $\mathrm{P}-\mathrm{RT}$ during marking time on sitting ranged from 0.84 to 0.94 . An ICC of 0.90 for marking time on sitting was achieved by the 3 rd time of measurement. The ICC of P-RT during marking time on standing ranged from 0.89 to 0.97 . An ICC of 0.96 for marking time on standing was achieved by the 2 nd time of measurement. The ICC of P-RT during walking ranged from 0.86 to 0.94 . An ICC of 0.92 for walking was achieved by the 2 nd time of measurement. The ICC of P-RT during tapping with the non-dominant hand at $1 \mathrm{~Hz}$ in synchrony with beats of metronome ranged from 0.92 to 0.97 . An ICC of 0.92 was achieved by the $1 \mathrm{st}$ measurement time. The ICC of tapping with the non-dominant hand at $4 \mathrm{~Hz}$ in synchrony with beats of metronome ranged from 0.49 to 0.94 . However, the ICC of P-RT during tapping with the nondominant hand at $4 \mathrm{~Hz}$ in synchrony with beats of metronome achieved a high value of 0.90 by the 4 th 
Table 2. ICC of Different Types of Repetitive Movements $(n=10)$

\begin{tabular}{ccccccl}
\hline Times of measurement & SRT & MSI & MST & W & $1 \mathrm{~Hz}$ & $4 \mathrm{~Hz}$ \\
\hline 1 & 0.52 & $0.91^{* *}$ & $0.89^{* *}$ & $0.86^{* *}$ & $0.92^{* *}$ & 0.49 \\
2 & $0.82^{* *}$ & $0.84^{* *}$ & $0.96^{* *}$ & $0.92^{* *}$ & $0.96^{* *}$ & $0.71^{*}$ \\
3 & $0.89^{* *}$ & $0.90^{* *}$ & $0.95^{* *}$ & $0.94^{* *}$ & $0.97^{* *}$ & $0.85^{* *}$ \\
4 & $0.92^{* *}$ & $0.93^{* *}$ & $0.96^{* *}$ & $0.92^{* *}$ & $0.96^{* *}$ & $0.90^{* *}$ \\
5 & $0.95^{* *}$ & $0.96^{* *}$ & $0.97^{* *}$ & $0.93^{* *}$ & $0.93^{* *}$ & $0.91^{* *}$ \\
6 & $0.97^{* *}$ & $0.95^{* *}$ & $0.96^{* *}$ & $0.92^{* *}$ & $0.97^{* *}$ & $0.94^{* *}$ \\
7 & $0.98^{* *}$ & $0.94^{* *}$ & $0.96^{* *}$ & $0.91^{* *}$ & $0.96^{* *}$ & $0.94^{* *}$ \\
8 & $0.98^{* *}$ & $0.95^{* *}$ & $0.97^{* *}$ & $0.90^{* *}$ & $0.95^{* *}$ & $0.92^{* *}$ \\
9 & $0.98^{* *}$ & $0.94^{* *}$ & $0.97^{* *}$ & $0.90^{* *}$ & $0.95^{* *}$ & $0.92^{* *}$ \\
10 & $0.98^{* *}$ & $0.94^{* *}$ & $0.97^{* *}$ & $0.90^{* *}$ & $0.94^{* *}$ & $0.93^{* *}$ \\
\hline
\end{tabular}

$* \mathrm{p}<0.05, * * \mathrm{p}<0.01$. Abbreviation of each movement is shown in Table 1 .

measurement time. Reliability analysis showed that all measurements of P-RT had high test-retest reliability in the different types of movement. The P-RT of the five movements showed higher reliability than the SRT. ICC of P-RT marking time on sitting, marking time on standing, walking, tapping with the non-dominant hand at $1 \mathrm{~Hz}$ in synchrony with beats of metronome showed high values from the 2 nd or the 3 rd time.

There was no significant difference between the means of each time from the 3 rd to the 9 th measurement time compared to the mean of 10 measurement times. The consistencies of P-RT between the mean of 10 time of measurement and each time of measurement are shown in Table 3. The consistency of P-RT between the mean of the 10th measurement and each time of measurement in the six different types of movement showed high significance $(\mathrm{p}<0.05)$.

\section{DISCUSSION}

The purpose of this study was to verify the reliability of the P-RT in different types of repetitive movements. The ICC of P-RT had high values compared to SRT, and had significant reliability in the different types of repetitive movements.

Another purpose of the study was to determine the minimal number of measurement time considered optimal when measuring P-RT in the different types of repetitive movement used in the experiment.

The results obtained in this study indicate that the minimal number of measurement time is 3 times (tapping at $4 \mathrm{~Hz}$ excluded) for the young healthy
Table 3. Consistency of P-RT (Comparison Between the Mean of Each Time and that of the 10th Time) $(n=10)$

\begin{tabular}{ccccccc}
\hline Times & SRT & MTSi & MTSt & W & $1 \mathrm{~Hz}$ & $4 \mathrm{~Hz}$ \\
\hline 1 & $0.87^{* *}$ & $0.98^{* *}$ & $0.89^{* *}$ & $0.96^{* *}$ & $0.94^{* *}$ & $0.89 * *$ \\
2 & $0.94 * *$ & $0.97^{* *}$ & $0.96^{* *}$ & $0.98^{* *}$ & $0.98^{* *}$ & $0.95^{* *}$ \\
3 & $0.95^{* *}$ & $0.98^{* *}$ & $0.98^{* *}$ & $0.99^{* *}$ & $0.99 * *$ & $0.98^{* *}$ \\
4 & $0.98^{* *}$ & $0.99^{* *}$ & $0.99^{* *}$ & $1.00^{* *}$ & $0.99^{* *}$ & $0.99^{* *}$ \\
5 & $0.99 * *$ & $1.00^{* *}$ & $0.99 * *$ & $1.00^{* *}$ & $0.99 * *$ & $0.99 * *$ \\
6 & $1.00^{* *}$ & $1.00^{* *}$ & $0.99 * *$ & $1.00^{* *}$ & $1.00^{* *}$ & $0.99 * *$ \\
7 & $1.00^{* *}$ & $1.00^{* *}$ & $0.99^{* *}$ & $1.00^{* *}$ & $1.00^{* *}$ & $0.99 * *$ \\
8 & $1.00^{* *}$ & $1.00^{* *}$ & $1.00^{* *}$ & $1.00^{* *}$ & $1.00^{* *}$ & $1.00^{* *}$ \\
9 & $1.00^{* *}$ & $1.00^{* *}$ & $1.00^{* *}$ & $1.00^{* *}$ & $1.00^{* *}$ & $1.00^{* *}$ \\
\hline
\end{tabular}

$*: \mathrm{p}<0.05, * *: \mathrm{p}<0.01$.

Abbreviation of each movement was shown in Table 1.

people. Our study confirmed that measurement times of P-RT differed among the various types of repetitive movement. The results of our study further confirm that the mean of the 3rd time of measurement was the minimal number of times for reliable measurement of P-RT in the different types of repetitive movements (tapping at $4 \mathrm{~Hz}$ excluded). Collectively these results suggest that consistency of P-RT of the $3 \mathrm{rd}$ time of measurement is approximately similar to that of the 10th time of measurement (Table 3), indicating that an optimal ICC for P-RT could be achieved at the mean of the $3 \mathrm{rd}$ time of measurement (tapping at $4 \mathrm{~Hz}$ excluded). The results also showed significant reliability among the different types of repetitive movement. Furthermore, the number of times of measurement of P-RT for optimal results could be different among various types of repetitive movement. The ICCs obtained in this study were high even though the limiting criterion of ICC was 
0.90 .

P-RT has been used in psychological tests, fall risk assessment and prevention, and also intervention in the treatment of patients in the clinical field. It is useful for the evaluation and treatment of people who could not able to walk, weak people, or the severely handicapped.

In summary, the results of this study show that the optimal measurement time is the 3 rd time, suggesting that it may not be necessary to test young subjects for 10 times. This would save the patients' energy and the time of testing, hence increasing efficiency and patients' satisfaction and compliance, while the results retain high reliability.

\section{REFERENCES}

1) Stephen RL, Hylton BM: A physiological profile approach to falls risk assessment and prevention. Phys Ther, 2003, 83: 237-253.

2) Faulkner KA, Redfern MS, Rosano C, et al.: Reciprocal influence of concurrent walking and cognitive testing on performance in older adults. Gaitpost, 2006, 24: 182-189.
3) William H, Ryan J: The allocation of attention during locomotion is altered by anxiety. Exp Brain Res, 2003, 150: 385-394.

4) Kurosawa $\mathrm{K}$, Maruyama $\mathrm{H}$ : Reaction Time and Gait in the Gait. 1st Edition. Tokyo: IPEC, 2003, pp79-86.

5) Sajiki N, Isagoda A, Moriai N, et al.: Reaction time during walking. Perceptual and Motor Skills, 1989, 69: 259-262.

6) Nakamura R, Amakusa B: Reaction time during stepping movement. Human Ergol, 1982, 11: 211-213.

7) Kurosawa K: Movement analysis, analysis based on the reaction time. Rigaku Ryoho No Tameno Undo Seiri, 1993, 8: 135-140.

8) Nagasaki H: Ability and Disability of the Body, Tokyo: Chuoukoron, 1997, pp39-40.

9) Lord SR, Ward JA, Williams P, et al.: Physiological factors associated with fall in older communitydwelling women. J Am Geriatr Soc, 1994, 42: 11101117.

10) Lundin-Olsson L, Nyberg L, Gustafson Y: "Stops walking when talking" as a predictor of fall in elderly people [letter]. Lancet, 1997, 349: 617.

11) Stephen RL, Richard CF: Choice stepping reaction time: a composite measure of falls risk in older people. J Gerontol Med Sci, 2001, 56: 627-632. 